\title{
Variational Iteration Method for Volterra Functional Integrodifferential Equations with Vanishing Linear Delays
}

\author{
Ali Konuralp and H. Hilmi Sorkun \\ Department of Mathematics, Faculty of Arts and Sciences, Celal Bayar University, Muradiye Campus, \\ Yunus Emre, 45140 Manisa, Turkey \\ Correspondence should be addressed to Ali Konuralp; ali.konuralp@cbu.edu.tr
}

Received 30 April 2014; Revised 29 June 2014; Accepted 29 June 2014; Published 15 July 2014

Academic Editor: Luigi Muglia

Copyright (C) 2014 A. Konuralp and H. H. Sorkun. This is an open access article distributed under the Creative Commons Attribution License, which permits unrestricted use, distribution, and reproduction in any medium, provided the original work is properly cited.

Application process of variational iteration method is presented in order to solve the Volterra functional integrodifferential equations which have multi terms and vanishing delays where the delay function $\theta(t)$ vanishes inside the integral limits such that $\theta(t)=q t$ for $0<q<1, t \geq 0$. Either the approximate solutions that are converging to the exact solutions or the exact solutions of three test problems are obtained by using this presented process. The numerical solutions and the absolute errors are shown in figures and tables.

\section{Introduction}

Nowadays, understanding also from the work of Brunner [1], we are faced with some important problems including the numerical analysis of Volterra functional equations with vanishing delays. He exposed open problems about numerical analysis of $k$ th-order Volterra functional integrodifferential equations (VFIDE):

$$
\begin{aligned}
u^{(k)}(t)= & \sum_{l=0}^{k-1} a_{l+1}(t) u^{(l)}(t)+\sum_{l=0}^{k-1} b_{l+1}(t) u^{(l)}(\theta(t))+g(t) \\
& +\sum_{l=0}^{k-1}\left(V^{l} u\right)(t)+\sum_{l=0}^{k-1}\left(V_{\theta}^{l} u\right)(t),
\end{aligned}
$$

where $t \in I(k \geq 1)$ for $l=0, \ldots, k-1$,

$$
\begin{gathered}
\left(V^{l} u\right)(t)=\int_{0}^{t} K_{0, l+1}(t, s) u^{(l)}(s) d s, \\
\left(V_{\theta}^{l} u\right)(t)=\int_{0}^{\theta(t)} K_{1, l+1}(t, s) u^{(l)}(s) d s,
\end{gathered}
$$

as well as the multidelay pantograph-type VFIDE in [1]. During 1990s, Brunner et al. [2] and Hu [3] introduced geometric mesh concept in collocation methods in order to obtain the collocation solutions of the problems. On the other hand, the pantograph differential equations are employed for their numerical solutions by using various methods such as Taylor matrix method [4], variational iteration method $[5,6]$, differential transform method [7], and methods in other papers [8-12]. In [5], the process of VIM is given for the first order multipantograph equations. In [6], the variational iteration method is applied to some examples in order to obtain the numerical or exact solutions of the multipantograph equation where the coefficient $a_{1}(t)$ of $u(t)$ is a constant and additionally the Lagrange multiplier is given for (1) without terms (2) and (3), that is, only for the extended multipantograph equation.

The variational iteration method which obtains the analytical or numerical solutions of a wide spectrum of differential equations, as well as integral equations, was proposed in the late 90 s by $\mathrm{He}$ [13-15] and has been used in hundreds of papers by many authors in order to solve the well-known famous equations and to show the effectiveness, straightness, and convergence of that powerful method [1620]. Furthermore, since it is a useful mathematical tool that ensures its reliability, the method has been developed according to the needs [21-24] and has also been extended 
for fractional differential and fractional integrodifferential equations [25-28].

The basic idea behind variational iteration method is to construct an iteration formula for the considered equation. After finding optimized Lagrange multiplier for constructed correction functional (i.e., iteration formula), the method takes into account that corrected iteration formula and starts to iterate with an initial function. In most cases the method provides exact solutions or the series form of exact solutions.

In addition to [28], in this paper, the procedure of the variational iteration method is presented for the Volterra functional integrodifferential equations with vanishing delays (1), where the Volterra integral terms are as in (2) and the delayed Volterra integral terms are as in (3); then this extended scheme is applied to three test problems for showing the applicability of the procedure and the convergent numerical solutions to the exact solutions. The numerical data for different parameter values are also given by tables and figures.

\section{The Application Process of Variational Iteration Method to VFIDE}

Now the process how to apply the method for (1) is given. Firstly, for the basic idea of the method, let us consider the following nonlinear equation:

$$
L u(t)+N u(t)=g(t),
$$

where $L$ is a linear operator, $N$ is a nonlinear operator, and $g(t)$ is a known analytical function. According to variational iteration method, we can construct the following correction functional:

$$
u_{n+1}(t)=u_{n}(t)+\int_{0}^{t} \lambda\left[L u_{n}(\tau)+N u_{n}(\tau)-g(\tau)\right] d \tau
$$

$$
\text { for } n \geq 0 \text {, }
$$

where $\lambda$ is a general Lagrange multiplier which can be identified by variational theory, $u_{0}(t)$ is an initial approximation with possible unknowns, and $\widetilde{u}_{n}$ is considered as restricted variation, that is, $\delta \tilde{u}_{n}=0[14]$.

Now the above idea can be extended as follows. Considering $k$ th-order Volterra functional integrodifferential equation (1) with (2) and (3), the correction functional according to relation (5) can be written as

$$
\begin{aligned}
u_{n+1}(t) & \\
= & u_{n}(t) \\
& +\int_{0}^{t} \lambda(\tau ; t)\left\{u_{n}^{(k)}(\tau)-\sum_{l=0}^{k-1} a_{l+1}(\tau) u_{n}^{(l)}(\tau)\right.
\end{aligned}
$$

$$
\begin{array}{r}
-\sum_{l=0}^{k-1} b_{l+1}(\tau) u_{n}^{(l)}(\theta(\tau))-g(\tau) \\
\left.-\sum_{l=0}^{k-1}\left(V^{l} u_{n}\right)(\tau)-\sum_{l=0}^{k-1}\left(V_{\theta}^{l} u_{n}\right)(\tau)\right\} d \tau \\
t \in I(k \geq 1),
\end{array}
$$

where except the name of the variable $t$ involved for $l=$ $0, \ldots, k-1\left(V^{l} u_{n}\right)(\tau)$ and $\left(V_{\theta}^{l} u_{n}\right)(\tau)$ are as in (2) and (3), respectively. In order to specify the iteration, the Lagrange multiplier has to be found. The form of the Lagrange multiplier that will be determined by transferring the derivation from $u$ to $\lambda$ can be either a linear or a nonlinear function of $\tau$ and $t$. Supposing all the functions on the right hand side of the $k$ th-order derivation in (6) as a function such that

$$
f=f\left(u^{(l)}(\tau), u^{(l)}(\theta(\tau)), g(\tau),\left(V^{l} u\right)(\tau),\left(V_{\theta}^{l} u\right)(\tau)\right),
$$

$$
0 \leq l \leq k-1
$$

the correction functional (6) can be written as

$$
\begin{array}{r}
u_{n+1}(t)=u_{n}(t) \\
+\int_{0}^{t} \lambda(\tau ; t)\left\{u_{n}^{(k)}(\tau)-f\left(u_{n}^{(l)}(\tau), u_{n}^{(l)}(\theta(\tau)),\right.\right. \\
g(\tau),\left(V^{l} u_{n}\right)(\tau), \\
\left.\left.\left(V_{\theta}^{l} u_{n}\right)(\tau)\right)\right\} d \tau
\end{array}
$$

for $t \in I k \geq 1, n \geq 0$. Making the above correction functional stationary and noticing that $\delta \tilde{f}=0$ which represents all variables to be restricted, we obtain

$$
\begin{array}{r}
\delta u_{n+1}(t) \\
=\delta u_{n}(t) \\
+\int_{0}^{t} \lambda(\tau, t)\left\{\delta u_{n}^{(k)}(\tau)-\delta \tilde{f}\left(u_{n}^{(l)}(\tau), u_{n}^{(l)}(\theta(\tau)),\right.\right. \\
g(\tau),\left(V^{l} u_{n}\right)(\tau), \\
\left.\left.\quad\left(V_{\theta}^{l} u_{n}\right)(\tau)\right)\right\} d \tau, \\
\delta u_{n+1}(t)=\delta u_{n}(t)+\int_{0}^{t} \lambda(\tau, t) u_{n}^{(k)}(\tau) d \tau .
\end{array}
$$

In order to find the conditions on $\lambda$, highest order derivative appearing in the integrand in (10) is transferred from $u$ to $\lambda$ and so (10) becomes

$$
\begin{aligned}
\delta u_{n+1}(t)= & \delta u_{n}(t)+\left.\lambda(\tau ; t) \delta u_{n}^{(k-1)}(\tau)\right|_{\tau=t} \\
& -\int_{0}^{t} \lambda^{\prime}(\tau ; t) u^{(k-1)}(\tau) d \tau .
\end{aligned}
$$


After repeating this process $k-1$ times, we will eventually obtain an expression which $u_{n}^{(l)}(0 \leq l \leq k-1)$ can be decomposed from and their coefficients yield the stationary conditions. Thus, as it is indicated by using the analogue way in [22], the Lagrange multiplier is calculated as the polynomial type

$$
\lambda(\tau ; t)=(-1)^{k} \frac{(\tau-t)^{k-1}}{(k-1) !} .
$$

Therefore, on account of (12) the iteration functional is

$$
\begin{aligned}
& u_{n+1}(t) \\
& =u_{n}(t) \\
& +(-1)^{k} \int_{0}^{t} \frac{(\tau-t)^{k-1}}{(k-1) !} \\
& \quad \times\left\{u_{n}^{(k)}(\tau)\right. \\
& \quad-f\left(\tau, u_{n}^{(l)}(\tau), u_{n}^{(l)}(\theta(\tau)), g(\tau),\right. \\
& \left.\left.\quad\left(V^{l} u_{n}\right)(\tau),\left(V_{\theta}^{l} u_{n}\right)(\tau)\right)\right\} d \tau,
\end{aligned}
$$

where $0 \leq l \leq k-1, t \in I, k \geq 1$, and $n \geq 0$.

Nevertheless, for $k=1$ the correction functional becomes

$u_{n+1}(t)$

$$
\begin{aligned}
& =u_{n}(t) \\
& +\int_{0}^{t} \lambda(\tau ; t)\left\{u_{n}^{\prime}(\tau)-f\left(\tau, u_{n}(\tau), u_{n}(\theta(\tau)), g(\tau),\right.\right. \\
& \\
& \left.\left.\quad\left(V^{0} u_{n}\right)(\tau),\left(V_{\theta}^{0} u_{n}\right)(\tau)\right)\right\} d \tau
\end{aligned}
$$

and Lagrange multiplier is evaluated as $\lambda(\tau ; t)=-1$ from (12). But it can also be chosen as a nonlinear function in order to accelerate the convergent rate of correction functional (14) so that the function $f$ can be modified as $f_{1}$ by excepting the function $u_{n}(\tau)$ with its coefficients. Now we have

$$
\begin{aligned}
& u_{n+1}(t) \\
& =u_{n}(t) \\
& +\int_{0}^{t} \lambda(\tau, t)\left\{u_{n}^{\prime}(\tau)-a_{1}(\tau) u_{n}(\tau)\right. \\
& -f_{1}\left(u_{n}(\theta(\tau)), g(\tau)\right. \\
& \left.\left.\quad\left(V^{0} u_{n}\right)(\tau),\left(V_{\theta}^{0} u_{n}\right)(\tau)\right)\right\} d \tau .
\end{aligned}
$$

Making (15) stationary and noticing that $\delta \widetilde{f}_{1}=0$, it is obviously obtained that

$$
\begin{gathered}
\delta u_{n+1}(t)=\delta u_{n}(t)+\int_{0}^{t} \lambda(\tau, t)\left(\delta u_{n}^{\prime}(\tau)-a_{1}(\tau) \delta u_{n}(\tau)\right) d \tau \\
\delta u_{n+1}(t)=\delta u_{n}(t)+\left.\lambda(\tau ; t) \delta u_{n}(\tau)\right|_{\tau=t} \\
-\int_{0}^{t}\left(\lambda^{\prime}(\tau ; t)+a_{1}(\tau) \lambda(\tau ; t)\right) \delta u_{n}(\tau) d \tau
\end{gathered}
$$

and then we have the following stationary conditions:

$$
\begin{gathered}
\delta u_{n}(t): 1+\left.\lambda(\tau ; t)\right|_{\tau=t}=0, \\
\delta u_{n}(\tau): \lambda^{\prime}(\tau ; t)+a_{1}(\tau) \lambda(\tau ; t)=0,
\end{gathered}
$$

which are the same conditions obtained in [5], so the Lagrange multiplier is found:

$$
\lambda(\tau ; t)=-e^{\int_{0}^{t} a_{1}(\xi) d \xi-\int_{0}^{\tau} a_{1}(\xi) d \xi}=-e^{\int_{\tau}^{t} a_{1}(\xi) d \xi} .
$$

Accordingly, the correction functional for $k=1$ is

$$
\begin{aligned}
& u_{n+1}(t) \\
& =u_{n}(t)-\int_{0}^{t} e^{\int_{\tau}^{t} a_{1}(\xi) d \xi} \\
& \times\left\{u_{n}^{\prime}(\tau)-a_{1}(\tau) u_{n}(\tau)-b_{1}(\tau) u_{n}(\theta(\tau))\right. \\
& \left.\quad-g(\tau)-\left(V^{0} u_{n}\right)(\tau)-\left(V_{\theta}^{0} u_{n}\right)(\tau)\right\} d \tau .
\end{aligned}
$$

By using formulae (13) and (19), with the given initial term $u_{0}$, the sequence $\left(u_{n}\right)$ is identified and the $n$th term of the sequence should be the approximate solution of problem (1).

\section{Numerical Examples}

In this section we show how the method can be applied to such problems so we give some examples that are the modifications of (1) for the numerical verification of the presented method in Section 2.

Example 1. Firstly, we have

$$
\begin{gathered}
\dot{u}(t)=u(t)+\left(t-t^{2}\right) u\left(\frac{t}{2}\right) \\
+\int_{0}^{t} t e^{-s} u(s) d s+\int_{0}^{t / 2}\left(t^{2}-2 s-2\right) u(s) d s, \\
u(0)=1,
\end{gathered}
$$

where the functions are considered as $k=1, a_{1}(t)=1, b_{1}(t)=$ $t-t^{2}, \theta(t)=t / 2, g(t)=0, K_{0,1}(t, s)=t e^{-s}, K_{1,1}(t, s)=t^{2}-$ $2 s-2$ in (1) with (2) and (3).

In order to solve the problem (20) by means of VIM, we use the proposed procedure in Section 2. Considering 
the nonlinear Lagrange multiplier (18), since $a_{1}(t)=1$, the Lagrange multiplier is directly calculated $\lambda(\tau ; t)=-e^{\int_{\tau}^{t} d \xi}=$ $-e^{t-\tau}$ so that the correction functional is

$$
\begin{aligned}
& u_{n+1}(t) \\
&=u_{n}(t)-\int_{0}^{t} e^{t-\tau}\left\{u_{n}^{\prime}(\tau)-u_{n}(\tau)-\left(\tau-\tau^{2}\right) u_{n}\left(\frac{\tau}{2}\right)\right. \\
&-\int_{0}^{\tau} \tau e^{-s} u_{n}(s) d s \\
&\left.-\int_{0}^{\tau / 2}\left(\tau^{2}-2 s-2\right) u_{n}(s) d s\right\} d \tau .
\end{aligned}
$$

Starting with $n=0$ and since the initial function is $u_{0}(t)=1$, from iteration formula (21)

$$
\begin{aligned}
u_{1}(t)=- & \frac{3}{2}+\frac{9}{4} e^{t}-\frac{3}{2} t-\frac{1}{4} t^{2}+\frac{1}{2} t e^{-t}+\frac{1}{4} e^{-t}-\frac{1}{2} t^{3} \\
u_{2}(t)= & \frac{125}{16}+\frac{61}{16} t-\frac{125}{16} t e^{-t}-\frac{73}{16} e^{-t} t^{2} \\
& +\frac{5}{36} t e^{-2 t}-\frac{11}{8} t^{3} e^{-t}+\frac{5}{108} e^{-2 t} \\
& -\frac{247}{864} e^{t}+\frac{11}{32} t^{3}-\frac{125}{32} e^{-t}-\frac{39}{32} t^{2} \\
& +\frac{1}{12} t^{2} e^{-2 t}-\frac{8}{3} e^{-(1 / 2) t}-\frac{1}{4} t^{4} e^{-t} \\
& +\frac{1}{128} t^{6}+\frac{41}{384} t^{4}-\frac{11}{960} t^{5}+e^{-(1 / 2) t} t^{2}+\frac{1}{3} t^{3} e^{-1 / 2}
\end{aligned}
$$

The other terms of the sequences $\left(u_{n}\right)$ can be found by using the iteration formula with the previous terms and for the large values of $t$, because the exact solution of (20) is $e^{t}, u_{n}(t)$ for $n=3,4,5$ have more terms than previous ones and are not necessary to write here, but we can give these limitations

$$
\begin{gathered}
\lim _{t \rightarrow \infty} \frac{u_{3}(t)}{e^{t}}=1.0912, \quad \lim _{t \rightarrow \infty} \frac{u_{4}(t)}{e^{t}}=1.0130, \\
\lim _{t \rightarrow \infty} \frac{u_{5}(t)}{e^{t}}=1.0007 .
\end{gathered}
$$

It is obviously seen that using this iteration formula (21), the approximate solution $u_{5}(t)$ of the problem (20) that is convergent to the exact solution even for the large values of $x$ is found in the beginning terms of the sequence $\left(u_{n}\right)$. Tables 1 and 2 show the values of solutions for comparison purposes and Figures 1 and 2 support the efficiency and the accuracy of the method.

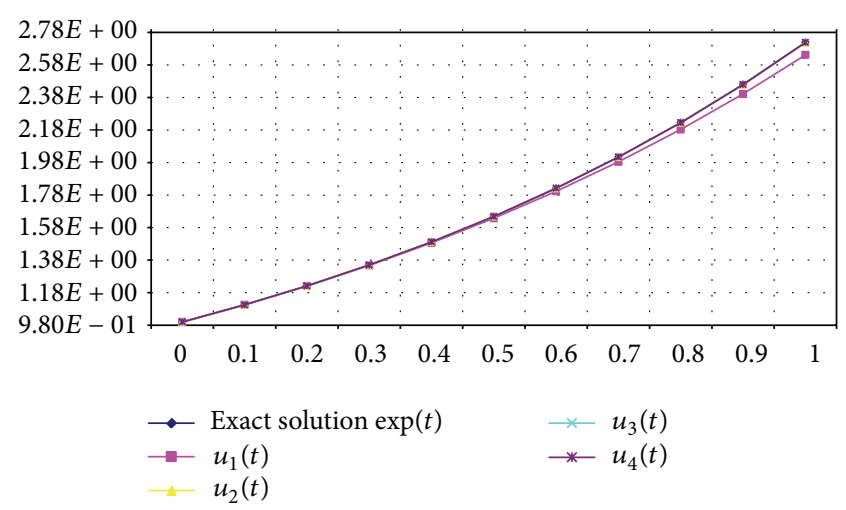

Figure 1: Approximate solutions $u_{i}(t) i=1,2,3,4$ and exact solution of Example 1.

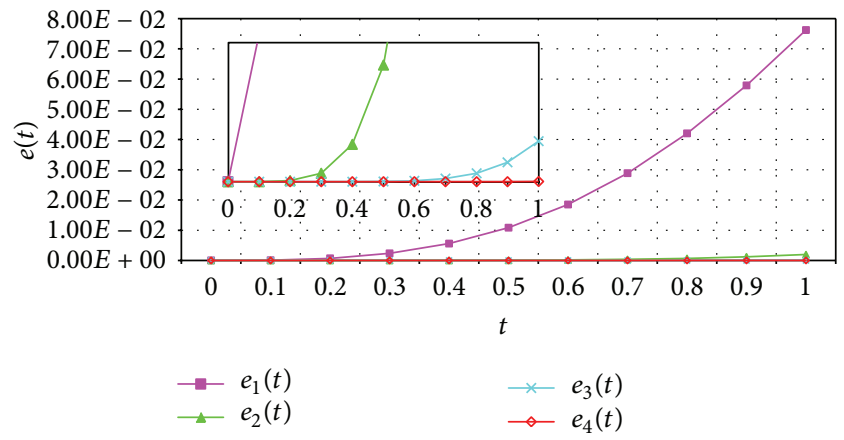

FIGURE 2: Comparison of errors of the approximate solutions where the absolute errors are $e_{i}(t)=\left|u(t)-u_{i}(t)\right|, i=1,2,3,4$.

Example 2. Now, we have

$$
\begin{gathered}
\dot{u}(t)=u(t)+u\left(\frac{t}{2}\right)-\frac{5}{3} t^{4}+4 t+2 \\
+\int_{0}^{t} 3 s u(s) d s+\int_{0}^{t / 2} 2 t u(s) d s \\
u(0)=-1,
\end{gathered}
$$

where the functions are considered as $k=1, a_{1}(t)=1$, $b_{1}(t)=1, \theta(t)=t / 2, g(t)=-(5 / 3) t^{4}+4 t+2, K_{0,1}(t, s)=3 s$, $K_{1,1}(t, s)=2 t$ in (1) with (2) and (3).

To solve the problem (24) by means of VIM, we consider the linear Lagrange multiplier evaluated from (12) for this problem. Since $k=1$, the Lagrange multiplier is directly calculated $\lambda(\tau ; t)=-1$ so that the correction functional is

$$
\begin{aligned}
u_{n+1}(t)= & u_{n}(t) \\
& -\int_{0}^{t}\left[u_{n}^{\prime}(\tau)-u_{n}(\tau)-u\left(\frac{\tau}{2}\right)+\frac{5}{3} \tau^{4}-4 \tau-2\right. \\
& \left.-\int_{0}^{\tau} 3 s u(s) d s-\int_{0}^{\tau / 2} 2 t u(s) d s\right] d \tau
\end{aligned}
$$


TABle 1: Numerical results of Example 1 for the approximate solution $u_{i}(t), i=1,2,3,4$.

\begin{tabular}{lccccc}
\hline$t$ & Exact solution $e^{t}$ & $u_{1}(t)$ & $u_{2}(t)$ & $u_{3}(t)$ & 1 \\
\hline 0.0 & 1 & 1 & 1 & $u_{4}(t)$ \\
0.1 & 1.1051709180756476248 & 1.1050857910809950277 & 1.1051709006185340780 & 1.1051709180747629519 & 1.1051709180753588641 \\
0.2 & 1.2214027581601698339 & 1.2207119694376757768 & 1.2214021515886701535 & 1.2214027580088591109 & 1.2214027581601910911 \\
0.3 & 1.3498588075760031040 & 1.3475096053186941304 & 1.3498539189718694827 & 1.3498588043837122657 & 1.3498588075750836373 \\
0.4 & 1.4918246976412703178 & 1.4862495904088959004 & 1.4918032466172082472 & 1.4918246696710125717 & 1.4918246976250816793 \\
0.5 & 1.6487212707001281468 & 1.6378881889316050424 & 1.6486542210674227886 & 1.6487211208887711166 & 1.6487212705453547801 \\
0.6 & 1.8221188003905089749 & 1.8036137007303597314 & 1.8219504259837133523 & 1.8221182162906284352 & 1.8221187994271196311 \\
0.7 & 2.0137527074704765216 & 1.9848947740834178825 & 2.0133904299751254578 & 2.0137508858816475851 & 2.0137527030209720537 \\
0.8 & 2.2255409284924676046 & 2.1835309157842461447 & 2.2248469229147350931 & 2.2255361192359802622 & 2.2255409120307558656 \\
0.9 & 2.4596031111569496638 & 2.4017057619215561218 & 2.4583899838348527939 & 2.4595919593157148094 & 2.4596030598388968997 \\
1.0 & 2.7182818284590452354 & 2.6420436949114335208 & 2.7163148376495047572 & 2.7182585386203368168 & 2.7182816889366104589 \\
\hline
\end{tabular}

as in (13). To be more accurate in finding the solution, it is obvious to start the initial function as a polynomial type of order two because of the structure of the equation in (24). Starting with $n=0$ and since the initial function is $u_{0}(t)=$ $a t^{2}+b t+c$, from iteration formula (25)

$$
\begin{aligned}
u_{1}(t)= & \left(-\frac{1}{3}+\frac{1}{6} a\right) t^{5}+\frac{5}{16} b t^{4}+\left(\frac{5}{6} c+\frac{5}{12} a\right) t^{3} \\
& +\left(2+\frac{3}{4} b\right) t^{2}+(2+2 c) t+c
\end{aligned}
$$

is found. From the condition $u(0)=-1, c=-1$ so (25) is

$$
\begin{aligned}
u_{1}(t)= & \left(-\frac{1}{3}+\frac{1}{6} a\right) t^{5}+\frac{5}{16} b t^{4}+\left(\frac{5}{12} a-\frac{5}{6}\right) t^{3} \\
& +\left(2+\frac{3}{4} b\right) t^{2}-1
\end{aligned}
$$

and because this must correspond to the initial polynomial function, $a=2, b=0$. Thus the first iteration solution of the problem (24) is (27) with substituting $a=2, b=0$ which is the exact solution.

Example 3. Finally, we have

$$
\begin{gathered}
\dot{u}(t)=-\frac{1}{2}\left(1+e^{-q t}\right)+\int_{0}^{t} u(s) d s-\frac{1}{2} \int_{0}^{q t} u(s) d s, \\
u(0)=1,
\end{gathered}
$$

where the functions are considered as $k=1, a_{1}(t)=0, b_{1}(t)=$ $0, \theta(t)=q t$ for $0<q<1, g(t)=-(1 / 2)\left(1+e^{-q t}\right), K_{0,1}(t, s)=$ $1, K_{1,1}(t, s)=-1 / 2$ in (1) with (2) and (3).

This example is different from other two examples and now it is not important which formula of the Lagrange multiplier will be used, because the coefficient $a_{1}(t)$ is zero and both of the linear form (12) and nonlinear form (18) give the desired multiplier as $\lambda(\tau ; t)=-1$ so that the correction functional is

$$
\begin{aligned}
u_{n+1}(t)=u_{n}(t)-\int_{0}^{t} & {\left[u_{n}^{\prime}(\tau)+\frac{1}{2}\left(1+e^{-q \tau}\right)\right.} \\
& \left.-\int_{0}^{\tau} u_{n}(s) d s+\frac{1}{2} \int_{0}^{q \tau} u_{n}(s) d s\right] d \tau .
\end{aligned}
$$

Starting with $n=0$ and since the initial function is $u_{0}(t)=1$, from iteration formula (29)

$$
\begin{aligned}
& u_{1}(t ; q)=1-\frac{2+2 q t-2 e^{-q t}-2 q t^{2}+q^{2} t^{2}}{4 q} \\
& u_{2}(t ; q) \\
& =1-\frac{2+2 q t-2 e^{-q t}-2 q t^{2}+q^{2} t^{2}}{4 q} \\
& +\left(24-48 q+12 q^{4} t^{2}+24 q^{2} t-24 t^{2} q^{3}\right. \\
& \quad-8 t^{3} q^{4}+48 q e^{-q t}+4 t^{4} q^{4}-2 q^{5} t^{4}+4 t^{3} q^{6} \\
& \left.\quad-24 e^{-q^{2} t}-2 q^{7} t^{4}+q^{8} t^{4}\right) \times\left(96 q^{4}\right)^{-1}
\end{aligned}
$$

are obtained. The other terms of the sequences $\left(u_{n}\right)$ can be found by using the iteration formula with substituting the previous terms. The fourth iteration solution $u_{4}(t ; q)$ coincides with the exact solution $e^{-t}$ of (28), and the approximate solution $u_{4}(t ; q)$ is changed infinitesimally by the parameter 


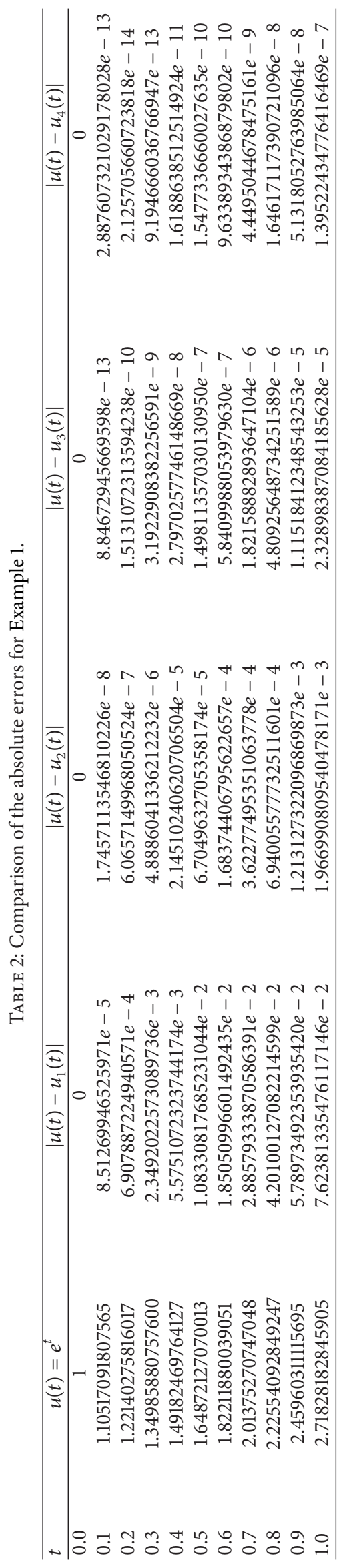




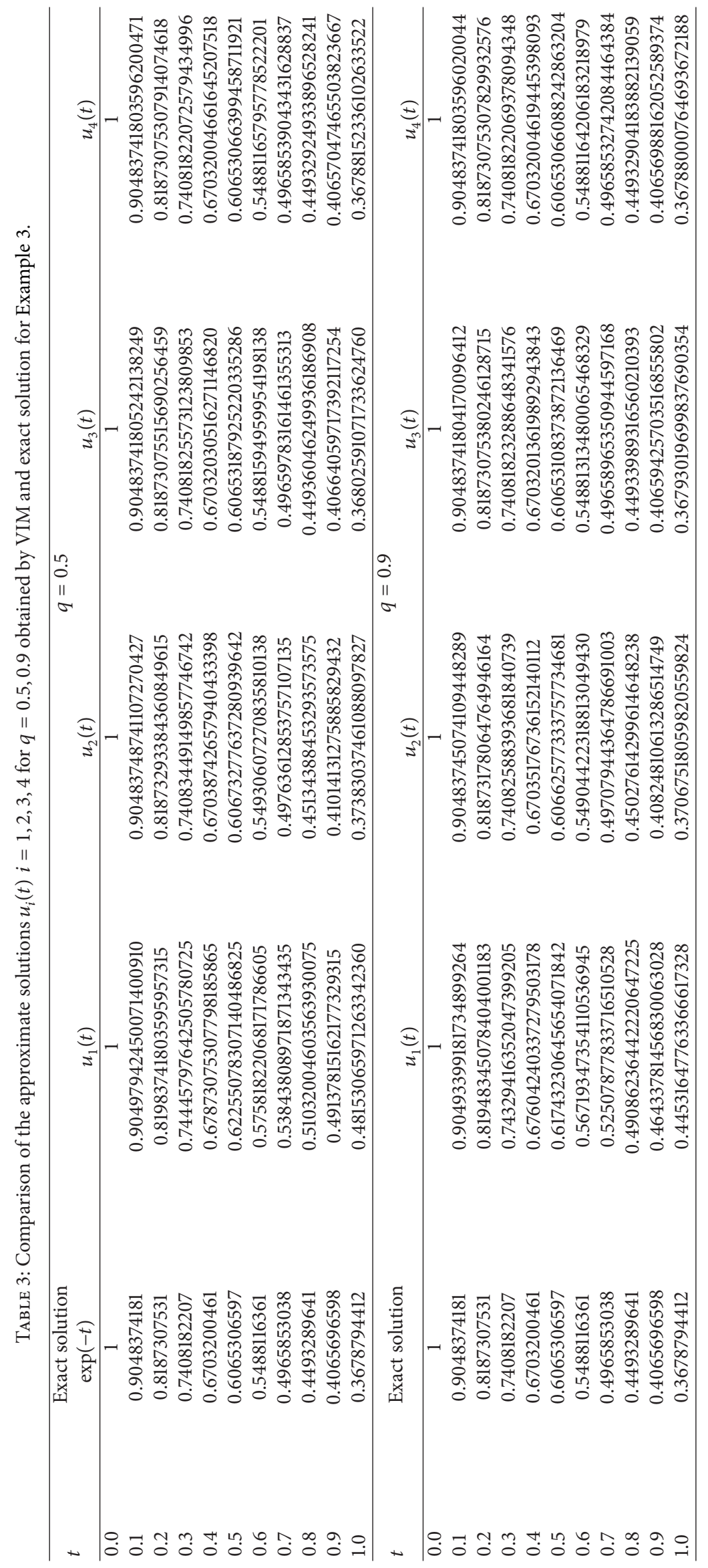




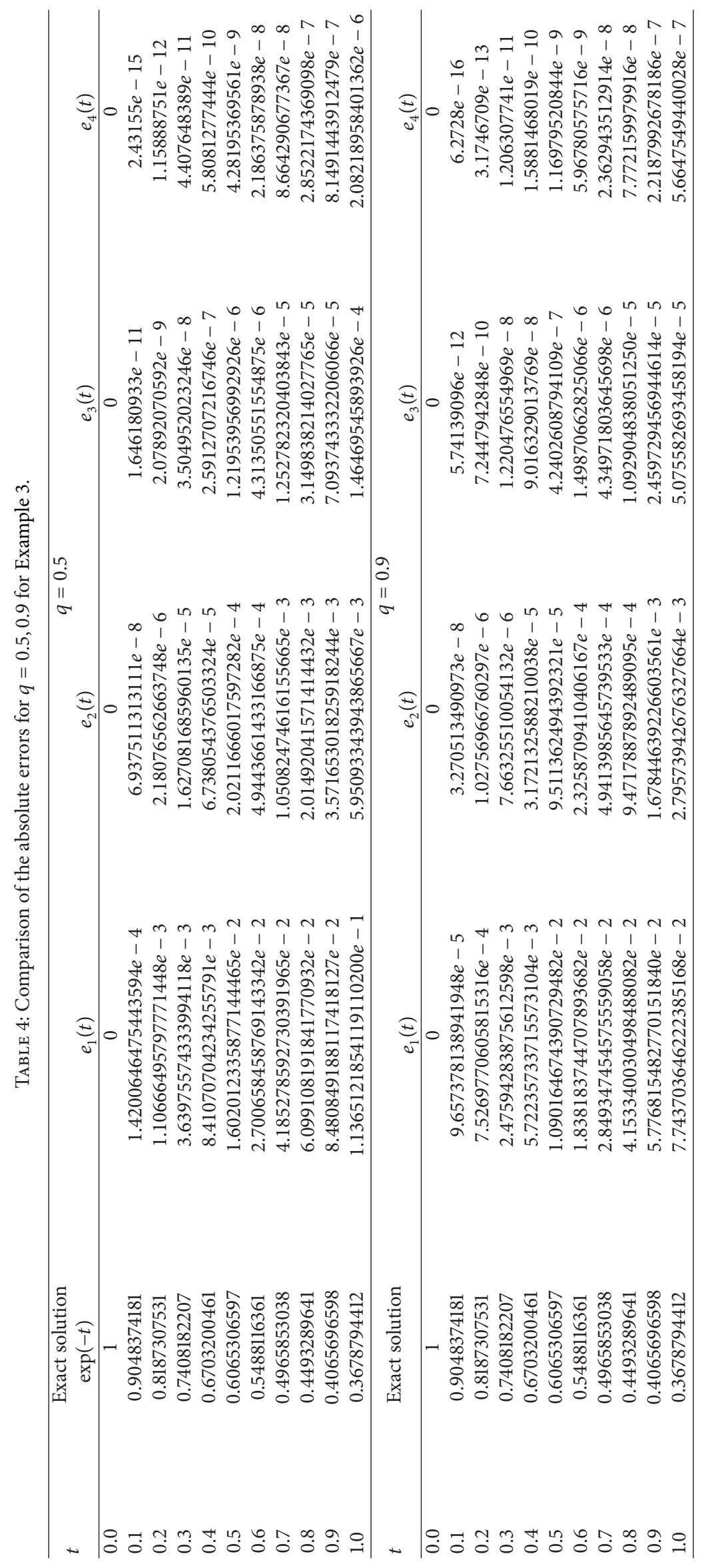




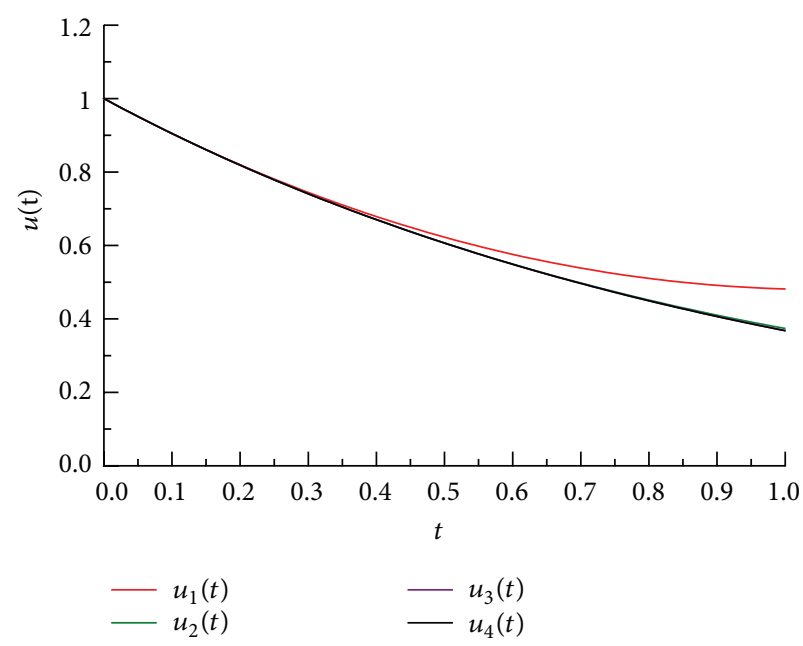

(a) For $q=0.5$

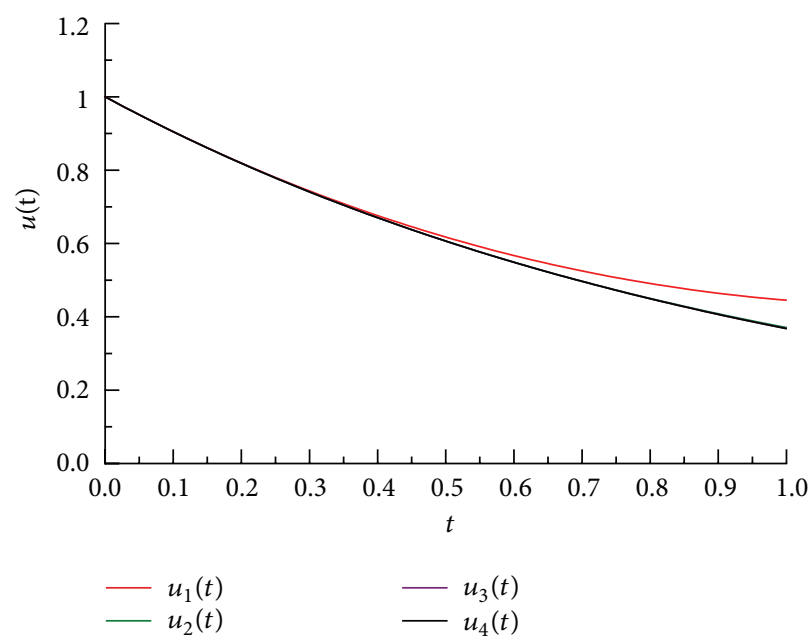

(b) For $q=0.9$

FIGURE 3: Comparison of approximate solutions for $q=0.5$ and $q=0.9$ in Example 3.

$q(0<q<1)$ in the $0 \leq t \leq 2$. For example, for $q=0.5$ the fourth iteration solution is

$$
\begin{aligned}
u_{4}(t)= & -0.5104166667 t^{3}-5.079365079 * 10^{-11} t^{4} \\
& +0.00001709908248 t^{8}+108.5 t \\
& +85 e^{-0.5 t}-4096 e^{-0.0625 t}+5440 e^{-0.125 t} \\
& -1428 e^{-0.25 t}-0.00008343590628 t^{7} \\
& +0.003531901041 t^{5}+5 * 10^{-13} t^{6}
\end{aligned}
$$

So it is clearly seen from Figure 3 that the fourth iteration solution is the approximate solution with the errors indicated in Tables 3 and 4.

\section{Conclusion}

In this study, the process of variational iteration method for the Volterra functional integrodifferential equations with vanishing delays (1), where the Volterra integral terms are as in (2), the delayed Volterra integral terms are as in (3), and $\theta(t)=q t$ for $0<q<1, t \geq 0$ is the linear delay function, is constructed and it is applied to the problems that are the different types of problem (1). In Section 2, two types of Lagrange multiplier are given, that is, linear one and nonlinear one. From also the previous papers $[19,26]$, it is understood that sometimes the nonlinear multiplier yields the more accurate approach than the linear one. The method is applicable also in the pantograph-type differential equations and Volterra integrodifferential equations with linear delay functions.

\section{Conflict of Interests}

The authors declare that there is no conflict of interests regarding the publication of this paper.

\section{References}

[1] H. Brunner, "Current work and open problems in the numerical analysis of Volterra functional equations with vanishing delays," Frontiers of Mathematics in China, vol. 4, no. 1, pp. 3-22, 2009.

[2] H. Brunner, Q. Hu, and Q. Lin, "Geometric meshes in collocation methods for Volterra integral equations with proportional time delays," Seminar für Angewandte mathematik 1999-25, Eidgenössische Technische Hochschule, Zürich, Switzerland, 1999.

[3] Q. Hu, "Geometric meshes and their application to Volterra integro-differential equations with singularities," IMA Journal of Numerical Analysis, vol. 18, no. 1, pp. 151-164, 1998.

[4] M. Sezer and S. Yalçinbaş, "Approximate solution of multipantograph equation with variable coefficients," Journal of Computational and Applied Mathematics, vol. 214, no. 2, pp. 406-416, 2008.

[5] Z.-H. Yu, "Variational iteration method for solving the multipantograph delay equation," Physics Letters A, vol. 372, no. 43, pp. 6475-6479, 2008.

[6] A. Saadatmandi and M. Dehghan, "Variational iteration method for solving a generalized pantograph equation," Computers \& Mathematics with Applications, vol. 58, no. 11-12, pp. 2190-2196, 2009.

[7] Y. Keskin, A. Kurnaz, M. E. Kiris, and G. Oturanc, "Approximate solutions of generalized pantograph equations by the differential transform method," International Journal of Nonlinear Sciences and Numerical Simulation, vol. 8, no. 2, pp. 159-164, 2007.

[8] Y. Liu, "Numerical investigation of the pantograph equation," Applied Numerical Mathematics, vol. 24, no. 2-3, pp. 309-317, 1997.

[9] H. Brunner, "The numerical analysis of pantograph-type Volterra functional integral equations," in Proceedings of the HKBU-NSYSU Workshop in Computational and Applied Mathematics, p. 6, Department of Mathematics, National Sun Yat-sen University, Hong Kong, April 2007.

[10] M. Z. Liu and D. Li, "Properties of analytic solution and numerical solution of multi-pantograph equation," Applied 
Mathematics and Computation, vol. 155, no. 3, pp. 853-871, 2004.

[11] G. Derfel and A. Iserles, "The pantograph equation in the complex plane," Journal of Mathematical Analysis and Applications, vol. 213, no. 1, pp. 117-132, 1997.

[12] S. Sedaghat, Y. Ordokhani, and M. Dehghan, "Numerical solution of the delay differential equations of pantograph type via Chebyshev polynomials," Communications in Nonlinear Science and Numerical Simulation, vol. 17, no. 12, pp. 4815-4830, 2012.

[13] J. He, "A new approach to nonlinear partial differential equations," Communications in Nonlinear Science and Numerical Simulation, vol. 2, no. 4, pp. 230-235, 1997.

[14] J. He, "Approximate solution of nonlinear differential equations with convolution product nonlinearities," Computer Methods in Applied Mechanics and Engineering, vol. 167, no. 1-2, pp. 69-73, 1998.

[15] J. He, "Variational iteration method for delay differential equations," Communications in Nonlinear Science and Numerical Simulation, vol. 2, no. 4, pp. 235-236, 1997.

[16] M. A. Abdou and A. A. Soliman, "Variational iteration method for solving BURger's and coupled BURger's equations," Journal of Computational and Applied Mathematics, vol. 181, no. 2, pp. 245-251, 2005.

[17] J. H. He, "Some asymptotic methods for strongly nonlinear equations," International Journal of Modern Physics B, vol. 20, no. 10, pp. 1141-1199, 2006.

[18] A.-M. Wazwaz, "The variational iteration method for exact solutions of Laplace equation," Physics Letters A, vol. 363, no. 4, pp. 260-262, 2007.

[19] A. Konuralp, "The steady temperature distributions with different types of nonlinearities," Computers \& Mathematics with Applications, vol. 58, no. 11-12, pp. 2152-2159, 2009.

[20] A. Barari, H. D. Kaliji, M. Ghadim, and G. Domairry, "Nonlinear vibration of Euler-Bernoulli beams," Latin American Journal of Solids and Structures, vol. 8, no. 2, pp. 139-148, 2011.

[21] M. Tatari and M. Dehghan, "On the convergence of He's variational iteration method," Journal of Computational and Applied Mathematics, vol. 207, no. 1, pp. 121-128, 2007.

[22] J. H. He and X. H. Wu, "Variational iteration method: new development and applications," Computers \& Mathematics with Applications, vol. 54, no. 7-8, pp. 881-894, 2007.

[23] J.-H. He, "Variational iteration method-some recent results and new interpretations," Journal of Computational and Applied Mathematics, vol. 207, no. 1, pp. 3-17, 2007.

[24] L. Xu, "Variational iteration method for solving integral equations," Computers \& Mathematics with Applications, vol. 54, no. 7-8, pp. 1071-1078, 2007.

[25] Z. M. Odibat and S. Momani, "Application of variational iteration method to nonlinear differential equations of fractional order," International Journal of Nonlinear Sciences and Numerical Simulation, vol. 7, no. 1, pp. 27-34, 2006.

[26] A. Konuralp, Ç. Konuralp, A. Yildirim, and A. Yıldırım, "Numerical solution to the Van der Pol equation with fractional damping," Physica Scripta, vol. 2009, Article ID 014034, 2009.

[27] X.-J. Yang and D. Baleanu, "Fractal heat conduction problem solved by local fractional variation iteration method," Thermal Science, vol. 17, no. 2, pp. 625-628, 2013.

[28] A. Konuralp and H. H. Sorkun, "The numerical solutions of volterra functional integro-differential equations having vanishing linear delays," in Proceedings of the 4th International Conference on Mathematical and Computational Applications, Manisa, Turkey, June 2013. 


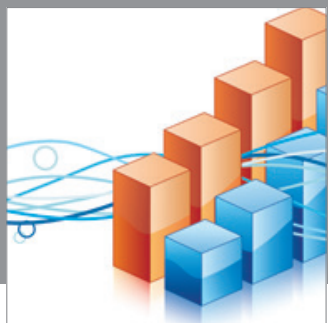

Advances in

Operations Research

mansans

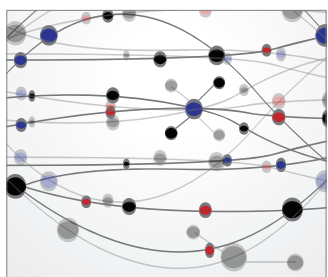

The Scientific World Journal
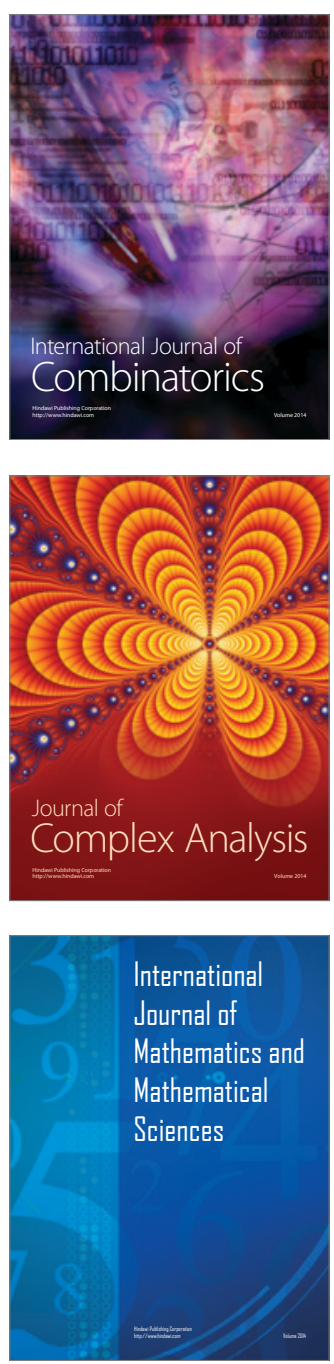
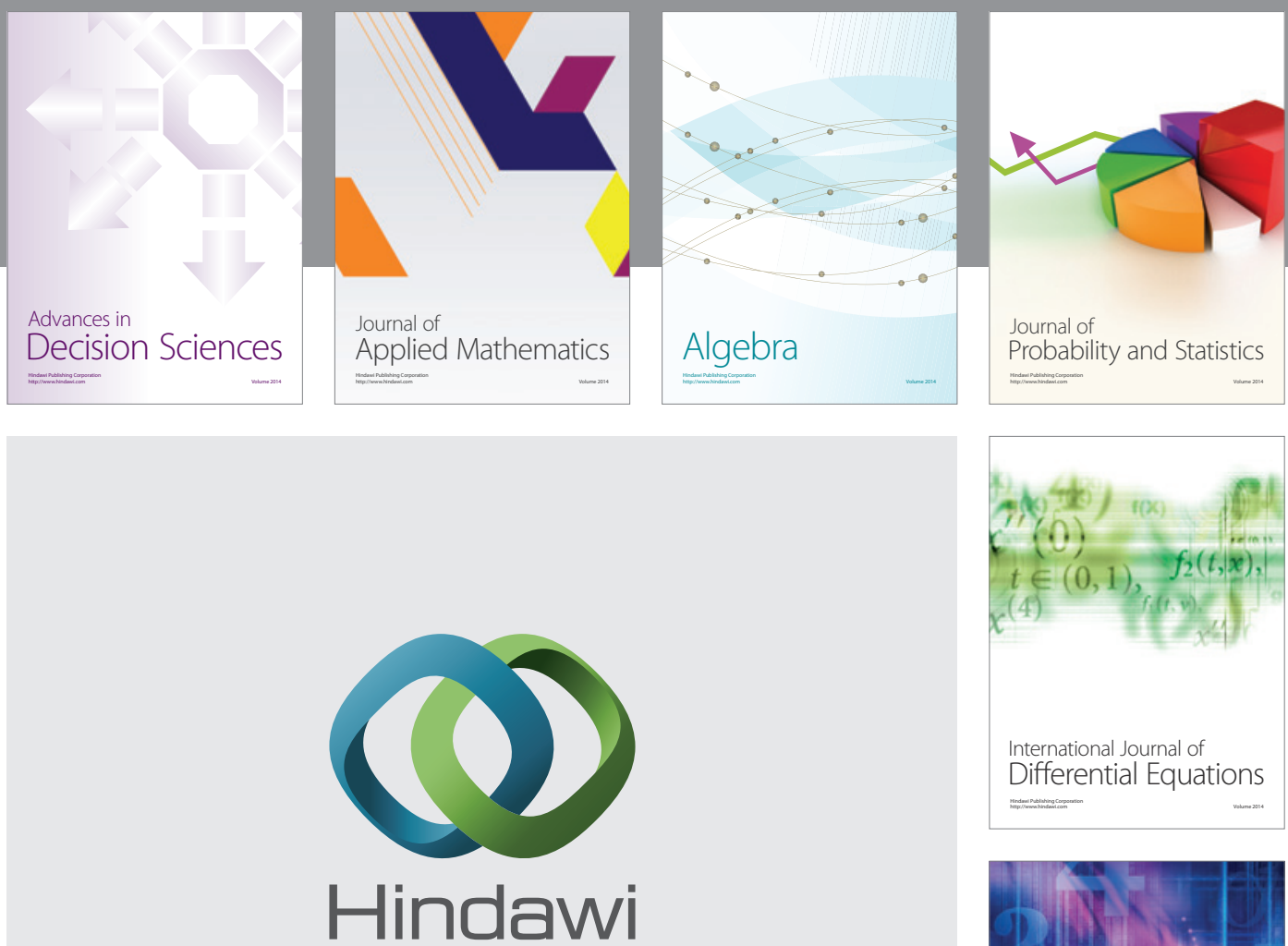

Submit your manuscripts at http://www.hindawi.com
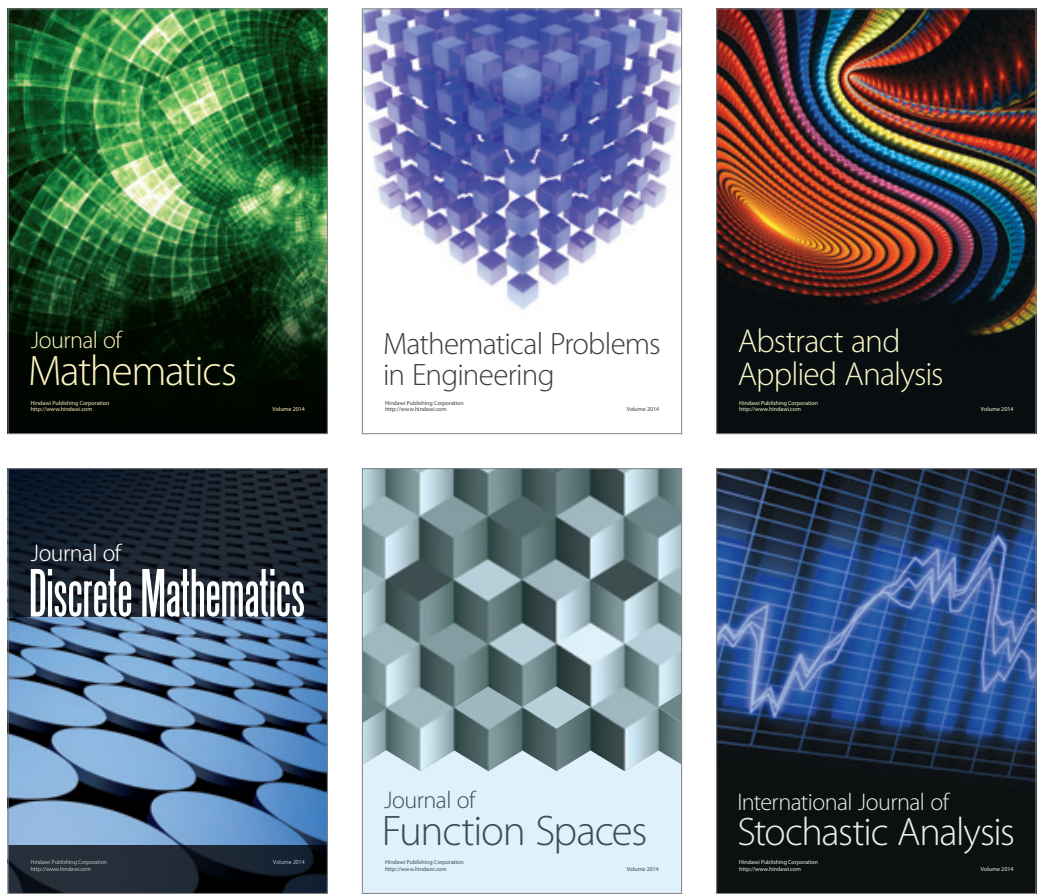

Journal of

Function Spaces

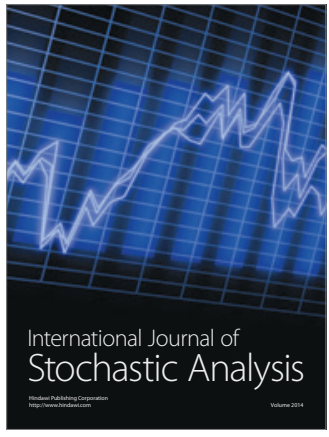

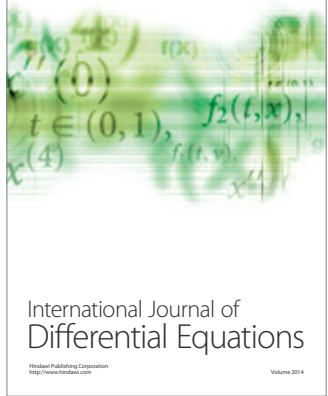
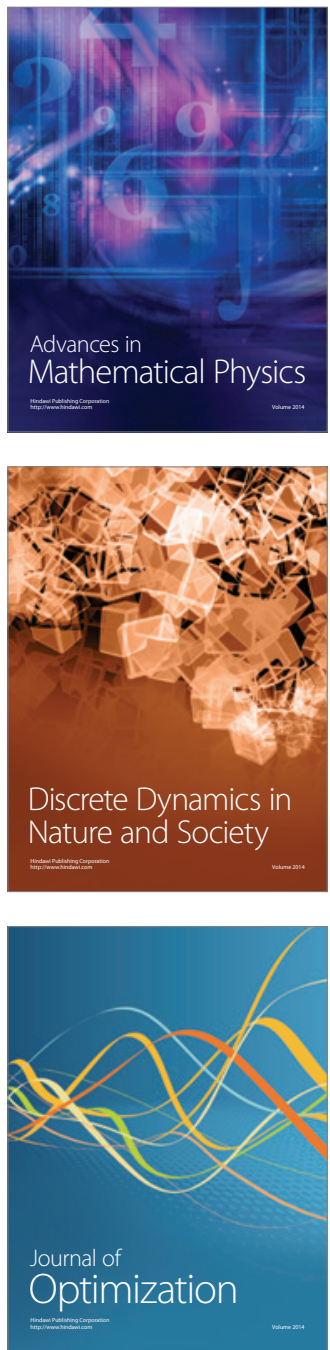